... manuscript No.

(will be inserted by the editor)

\title{
Homogeneous Riemannian structures on some solvable extensions of the Heisenberg group
}

\author{
W. Batat · P. M. Gadea · J. A. Oubiña
}

Received: date / Accepted: date

\begin{abstract}
Two families of four or five-dimensional Riemannian solvable Lie groups, which are extensions of the 3-dimensional Heisenberg group, are considered. We determine all the homogeneous Riemannian structures on them, and the simply connected groups of isometries corresponding to the associated reductive decompositions. Some of these structures are homogeneous Kähler or homogeneous cosymplectic, and in these cases they are realized by the complex hyperbolic plane $\mathbb{C H}(2)$ and by $\mathbb{C H}(2) \times \mathbb{R}$, respectively.
\end{abstract}

Keywords Homogeneous Riemannian structures - Solvable Riemannian Lie groups . Heisenberg group $\cdot$ Hermitian manifolds $\cdot$ Almost contact metric manifolds

Mathematics Subject Classification (2000) $53 \mathrm{C} 30 \cdot 53 \mathrm{C} 25 \cdot 53 \mathrm{C} 55$

\section{Introduction and preliminaries}

Ambrose and Singer extended in [3] to the homogeneous case the classical characterization by Cartan [6] of Riemannian symmetric spaces as those (connected) simply connected and complete Riemannian manifolds with parallel curvature tensor, in terms of a tensor field $S$ on the manifold satisfying certain properties (see (1.1) below). This tensor $S$ is called a homogeneous Riemannian structure by Tricerri and Vanhecke in [24], where a classification

The three authors have been partially supported by the Ministry of Science and Innovation, Spain, under Project MTM2011-22528.

W. Batat

École Normale Supérieure d'Enseignement Technologique d'Oran, Département de Mathématiques et Informatique, B.P. 1523, El M'Naouar, Oran, Algeria

E-mail: wafa.batat@enset-oran.dz

P. M. Gadea

Instituto de Física Fundamental, CSIC, Serrano 113-bis, 28006-Madrid, Spain

E-mail: pmgadea@iff.csic.es

J. A. Oubiña

Departamento de Xeometría e Topoloxía, Facultade de Matemáticas, Universidade de Santiago de Compostela, 15782-Santiago de Compostela, Spain

Tel.: +34881813141, Fax: +34981597054

E-mail: ja.oubina@usc.es 
of these structures was also given. The similar characterization for homogeneous almost Hermitian manifolds was obtained by Sekigawa in [23], and Abbena and Garbiero obtained a classification of homogeneous Kähler structures in [1]. The corresponding odd-dimensional version (the homogeneous almost contact metric structures), have been also studied (see [11, 12, 17, 20]).

Homogeneous Riemannian structures have shown to be useful in the study of the geometry of homogeneous Riemannian spaces and in particular to determine the groups of isometries (or holomorphic isometries, or isometries which leave invariant an almost contact structure, or even isometries which leave invariant a quaternionic structure) acting transitively on these spaces (see, for instance, [7-10,14, 15, 17]).

In this paper we consider two families of four or five-dimensional simply connected Riemannian Lie groups, which we denote by $\mathcal{A}_{4}(\lambda, \mu)$ and $\mathcal{A}_{5}(\lambda, \mu, v)$, respectively, where $\lambda, \mu, v$ are real numbers, $\lambda, \mu>0$. The groups $\mathcal{A}_{4}(\lambda, \mu)$ are all isomorphic, and they can be considered as the same Lie group but with different left-invariant Riemannian metrics $g_{\lambda, \mu}$. The groups $\mathcal{A}_{5}(\lambda, \mu, v)$ are not in general isomorphic. All these groups are solvable extensions of the Heisenberg group $H_{3}$ such that $H_{3}$ is a normal subgroup of all of them. If $v \neq 0$, each group $\mathcal{A}_{5}(\lambda, \mu, v)$ is a semidirect product $H_{3} \rtimes E$, where $E$ is an isomorphic copy of the affine group of the line. The present study of these spaces is motivated by the important role played by some of them, under certain relations between the parameters, in the Alekseevsky's classification [2] of homogeneous Einstein spaces with nonpositive curvature and dimension $n \leq 5$. For each $k>0$, the Ricci curvature tensor of $\mathcal{A}_{4}\left(\frac{\sqrt{6}}{3} k, \frac{\sqrt{6}}{6} k\right)$ and $\mathcal{A}_{5}\left(\frac{\sqrt{6}}{3} k, \frac{2 \sqrt{33}}{33} k, \frac{\sqrt{33}}{11} k\right)$ is of the form Ric $=-k^{2} g$, where $g$ is the metric, and the latter is the unique nonsymmetric space in that classification with this property.

The aim of the present paper is to obtain the homogeneous Riemannian structures on $\mathcal{A}_{4}(\lambda, \mu)$ and $\mathcal{A}_{5}(\lambda, \mu, v)$, their associated reductive decompositions and the corresponding simply connected groups of isometries. We also consider each $\mathcal{A}_{4}(\lambda, \mu)$ as a Hermitian homogeneous space, which is Kähler symmetric if $\lambda=2 \mu$, and each $\mathcal{A}_{5}(\lambda, \mu, v)$ as a homogeneous almost contact metric manifold, which is a cosymplectic symmetric space for $\lambda=2 \mu$ and $v=0$. After some preliminaries, Sections 2 and 3 are devoted to the metric Lie groups $\mathcal{A}_{4}(\lambda, \mu)$ and $\mathcal{A}_{5}(\lambda, \mu, v)$, respectively.

\subsection{Ambrose-Singer equations}

Let $(M, g)$ be a connected Riemannian manifold, and let $\nabla$ be the Levi-Civita connection of $g$. We adopt for the curvature tensor field $R$ of $\nabla$ the conventions

$$
R_{X Y} Z=\nabla_{[X, Y]} Z-\nabla_{X} \nabla_{Y} Z+\nabla_{Y} \nabla_{X} Z, \quad R_{X Y Z W}=g\left(R_{X Y} Z, W\right),
$$

for all $X, Y, Z, W \in \mathfrak{X}(M)$.

A simply connected and complete Riemannian manifold $(M, g)$ is Riemannian homogeneous if and only if there exists a $(1,2)$ tensor field $S$ on $M$ such that the connection $\widetilde{\nabla}=\nabla-S$ satisfies (see $[3,24]$ ) the Ambrose-Singer equations

$$
\widetilde{\nabla} g=0, \quad \widetilde{\nabla} R=0, \quad \widetilde{\nabla} S=0 .
$$

Such a structure $S$ is called a homogeneous Riemannian structure.

If a Riemannian manifold $(M, g)$ is homogeneous then $M=G / H$, where $G$ is a connected Lie group acting transitively and effectively on $M$ via isometries and $H$ is the isotropy 
subgroup at a base point $o \in M$. Then the Lie algebra $\mathfrak{g}$ of $G$ may be decomposed into a vector space direct sum $\mathfrak{g}=\mathfrak{h}+\mathfrak{m}$, where $\mathfrak{h}$ is the Lie algebra of $H$ and $\mathfrak{m}$ is an $\operatorname{Ad}(H)$-invariant subspace, i.e., $\operatorname{Ad}(H) \mathfrak{m} \subset \mathfrak{m}$. As $G$ is connected and $M$ simply connected, $H$ is connected, and the latter condition is equivalent to $[\mathfrak{h}, \mathfrak{m}] \subset \mathfrak{m}$. The vector space $\mathfrak{m}$ is identified with $T_{o}(M)$ under the isomorphism $X \in \mathfrak{m} \rightarrow \bar{X}_{o} \in T_{o}(M)$, where $\bar{X}$ is the Killing vector field on $M$ generated by the one-parameter subgroup $\{\exp t X\}$ of $G$ acting on $M$. The canonical connection $\widetilde{\nabla}$ of $M=G / H$ (with regard to the decomposition $\mathfrak{g}=\mathfrak{h}+\mathfrak{m}$ ) is determined by

$$
\left(\widetilde{\nabla}_{\bar{X}} \bar{Y}\right)_{o}=-\left(\overline{[X, Y]_{\mathfrak{m}}}\right)_{o}, \quad X, Y \in \mathfrak{m},
$$

and $S=\nabla-\widetilde{\nabla}$ satisfies (1.1) and it is a homogeneous Riemannian structure on $(M, g)$.

Conversely, let $S$ be a homogeneous Riemannian structure on a simply connected and complete Riemannian manifold $(M, g)$, and let $\widetilde{R}$ be the curvature tensor of the connection $\widetilde{\nabla}=\nabla-S$. We fix a point $o \in M$ and put $\mathfrak{m} \equiv T_{o} M$. The holonomy algebra $\tilde{\mathfrak{h}}$ of $\widetilde{\nabla}$ is the Lie subalgebra of the Lie algebra of skew-symmetric endomorphisms of $\left(\mathfrak{m}, g_{o}\right)$ generated by the operators $\widetilde{R}_{X Y}$, where $X, Y \in \mathfrak{m}$. Then (Nomizu [22], see also [3,24]), a Lie bracket is defined in the vector space direct sum $\tilde{\mathfrak{g}}=\tilde{\mathfrak{h}}+\mathfrak{m}$ by

$$
[V, W]=V W-W V, \quad[V, X]=V(X), \quad[X, Y]=\widetilde{R}_{X Y}+S_{X} Y-S_{Y} X,
$$

for all $V, W \in \tilde{\mathfrak{h}}, X, Y \in \mathfrak{m}$, and $\tilde{\mathfrak{g}}=\tilde{\mathfrak{h}}+\mathfrak{m}$ is said to be the reductive decomposition associated to the homogeneous Riemannian structure $S$. The simply connected Lie group $\widetilde{G}$ generated by $\tilde{\mathfrak{g}}$ acts transitively on $M$ via isometries and $M \equiv \widetilde{G} / \widetilde{H}$, where $\widetilde{H}$ is the connected Lie subgroup of $\widetilde{G}$ generated by $\tilde{\mathfrak{h}}$. The set $\Gamma$ of elements of $\widetilde{G}$ which act trivially on $M$ is a discrete normal subgroup of $\widetilde{G}$, and the Lie group $G=\widetilde{G} / \Gamma$ acts transitively and effectively on $M$ as a group of isometries, with isotropy subgroup $H=\widetilde{H} / \Gamma$. Then $M$ is diffeomorphic to $G / H$.

Now, if $S$ is a homogeneous Riemannian structure on a Riemannian manifold $(M, g)$, we also denote by $S$ the associated tensor field of type $(0,3)$ on $M$ defined by $S_{X Y Z}=g\left(S_{X} Y, Z\right)$. Then, the condition $\widetilde{\nabla} g=0$ in (1.1) is equivalent to $S_{X Y Z}=-S_{X Z Y}$ for all vector fields $X, Y, Z$ on $M$. Moreover, $\widetilde{\nabla} R=0$ is equivalent to the condition

$$
\left(\nabla_{Z} R\right)_{X_{1} X_{2} X_{3} X_{4}}=-R_{S_{Z} X_{1} X_{2} X_{3} X_{4}}-R_{X_{1}} S_{Z} X_{2} X_{3} X_{4}-R_{X_{1} X_{2} S_{Z} X_{3} X_{4}}-R_{X_{1} X_{2} X_{3} S_{Z} X_{4}}
$$

for all $Z, X_{1}, X_{2}, X_{3}, X_{4} \in \mathfrak{X}(M)$.

\section{A solvable one-dimensional extension of the Heisenberg group}

For each pair of positive real numbers $\lambda, \mu$, we denote by $\mathfrak{a}_{4}(\lambda, \mu)$ the metric Lie algebra with orthonormal basis $\{X, Y, P, Q\}$ and nonzero brackets

$$
[X, Y]=\lambda P, \quad[Q, X]=\mu X, \quad[Q, Y]=\mu Y, \quad[Q, P]=2 \mu P .
$$

Then $\mathfrak{a}_{4}(\lambda, \mu)$ is a solvable non-nilpotent Lie algebra that is the semidirect product of the Heisenberg algebra $\mathfrak{h}_{3}=\operatorname{Span}\{X, Y, P\}$, with $[X, Y]=\lambda P$, and the line $\mathfrak{q}$ generated by $Q$ under the homomorphism $\operatorname{ad}_{\mid \mathfrak{q}}: \mathfrak{q} \rightarrow \operatorname{Der}\left(\mathfrak{h}_{3}\right)$. We consider the Heisenberg group $H_{3}$ as $\mathbb{C} \times \mathbb{R}$ with the operation given by

$$
(z, p)\left(z^{\prime}, p^{\prime}\right)=\left(z+z^{\prime}, p+p^{\prime}+\frac{\lambda}{2} \operatorname{Im}\left(\bar{z} z^{\prime}\right)\right)
$$


so that the exponential map is exp: $x X+y Y+p P \in \mathfrak{h}_{3} \rightarrow((x+\mathrm{i} y), p) \in H_{3}$.

We denote by $\mathcal{A}_{4}(\lambda, \mu)$ the Riemannian, or metric, simply connected Lie group generated by $\mathfrak{a}_{4}(\lambda, \mu)$. As a manifold, it can be realized as $\mathbb{C} \times \mathbb{R}^{2}$, and parametrized by the global diffeomorphism

$$
(x, y, p, q) \in \mathbb{R}^{4} \equiv \mathfrak{a}_{4}(\lambda, \mu) \longrightarrow \exp (x X+y Y+p P) \exp (q Q) \in \mathcal{A}_{4}(\lambda, \mu) .
$$

With respect to these coordinates, and writing $z=x+\mathrm{i} y$, the group operation is given by

$$
(z, p, q) \cdot\left(z^{\prime}, p^{\prime}, q^{\prime}\right)=\left(z+\mathrm{e}^{\mu q} z^{\prime}, p+\mathrm{e}^{2 \mu q} p^{\prime}+\frac{\lambda}{2} \mathrm{e}^{\mu q} \operatorname{Im}\left(\bar{z} z^{\prime}\right), q+q^{\prime}\right),
$$

and the Lie group exponential map exp: $\mathfrak{a}_{4}(\lambda, \mu) \rightarrow \mathcal{A}_{4}(\lambda, \mu)$ is given by

$$
\exp (x X+y Y+p P+q Q)= \begin{cases}\left(\frac{e^{\mu q}-1}{\mu q}(x+\mathrm{i} y), \frac{e^{2 \mu q}-1}{2 \mu q} p, q\right) & \text { if } q \neq 0, \\ (x+\mathrm{i} y, p, 0) & \text { if } q=0 .\end{cases}
$$

The metric on $\mathcal{A}_{4}(\lambda, \mu)$ is the left-invariant Riemannian metric defined by the scalar product $\langle$,$\rangle on \mathfrak{a}_{4}(\lambda, \mu)$ and it is given by

$$
\begin{aligned}
g_{\lambda, \mu}=\mathrm{e}^{-4 \mu q}\left\{\left(\mathrm{e}^{2 \mu q}+\frac{\lambda^{2}}{4} y^{2}\right) \mathrm{d} x^{2}+\left(\mathrm{e}^{2 \mu q}\right.\right. & \left.+\frac{\lambda^{2}}{4} x^{2}\right) \mathrm{d} y^{2}+\mathrm{d} p^{2} \\
& \left.-\frac{\lambda^{2}}{2} x y \mathrm{~d} x \mathrm{~d} y+\lambda(y \mathrm{~d} x \mathrm{~d} p-x \mathrm{~d} y \mathrm{~d} p)\right\}+\mathrm{d} q^{2} .
\end{aligned}
$$

We notice that, for each $(\lambda, \mu)$ and $\left(\lambda^{\prime}, \mu^{\prime}\right)$, the corresponding Lie groups are isomorphic, but the metrics $g_{\lambda, \mu}$ and $g_{\lambda^{\prime}, \mu^{\prime}}$ coincide if and only if $(\lambda, \mu)=\left(\lambda^{\prime}, \mu^{\prime}\right)$.

The Levi-Civita connection on $\mathcal{A}_{4}(\lambda, \mu)$ is given by the Koszul formula $2\left\langle\nabla_{A} B, C\right\rangle=$ $\langle[A, B], C\rangle-\langle[B, C], A\rangle+\langle[C, A], B\rangle$ for all $A, B, C \in \mathfrak{a}_{4}(\lambda, \mu)$, and we have

$$
\begin{aligned}
& \nabla_{X} X=\mu Q, \quad \nabla_{Y} X=-\frac{\lambda}{2} P, \quad \nabla_{P} X=-\frac{\lambda}{2} Y, \quad \nabla_{Q} X=0, \\
& \nabla_{X} Y=\frac{\lambda}{2} P, \quad \nabla_{Y} Y=\mu Q, \quad \nabla_{P} Y=\frac{\lambda}{2} X, \quad \nabla_{Q} Y=0, \\
& \nabla_{X} P=-\frac{\lambda}{2} Y, \quad \nabla_{Y} P=\frac{\lambda}{2} X, \quad \nabla_{P} P=2 \mu Q, \quad \nabla_{Q} P=0, \\
& \nabla_{X} Q=-\mu X, \quad \nabla_{Y} Q=-\mu Y, \quad \nabla_{P} Q=-2 \mu P, \quad \nabla_{Q} Q=0 .
\end{aligned}
$$

Let $\left\{X^{*}, Y^{*}, P^{*}, Q^{*}\right\}$ be the basis of invariant 1-forms dual to $\{X, Y, P, Q\}$. The curvature tensor of $\nabla$ is given by

$$
\begin{aligned}
& R_{X Y}=\left(\frac{3 \lambda^{2}}{4}+\mu^{2}\right)\left(Y^{*} \otimes X-X^{*} \otimes Y\right)+\lambda \mu\left(P^{*} \otimes Q-Q^{*} \otimes P\right), \\
& R_{X P}=\left(\frac{\lambda^{2}}{4}-2 \mu^{2}\right)\left(X^{*} \otimes P-P^{*} \otimes X\right)+\frac{\lambda \mu}{2}\left(Y^{*} \otimes Q-Q^{*} \otimes Y\right), \\
& R_{X Q}=\mu^{2}\left(Q^{*} \otimes X-X^{*} \otimes Q\right)+\frac{\lambda \mu}{2}\left(P^{*} \otimes Y-Y^{*} \otimes P\right), \\
& R_{Y P}=\frac{\lambda \mu}{2}\left(Q^{*} \otimes X-X^{*} \otimes Q\right)+\left(\frac{\lambda^{2}}{4}-2 \mu^{2}\right)\left(Y^{*} \otimes P-P^{*} \otimes Y\right), \\
& R_{Y Q}=\frac{\lambda \mu}{2}\left(X^{*} \otimes P-P^{*} \otimes X\right)+\mu^{2}\left(Q^{*} \otimes Y-Y^{*} \otimes Q\right), \\
& R_{P Q}=\lambda \mu\left(X^{*} \otimes Y-Y^{*} \otimes X\right)+4 \mu^{2}\left(Q^{*} \otimes P-P^{*} \otimes Q\right) .
\end{aligned}
$$


2.1 Homogeneous Riemannian structures on $\mathcal{A}_{4}(\lambda, \mu)$

With the previous notations and definitions we have

Theorem 2.1 (a) If $\lambda \neq 2 \mu$, all the homogeneous Riemannian structures on $\mathcal{A}_{4}(\lambda, \mu)$ are given by

$$
\begin{aligned}
S= & \theta \otimes\left(X^{*} \wedge Y^{*}\right)+2 \mu P^{*} \otimes\left(P^{*} \wedge Q^{*}\right)-\frac{\lambda}{2} Y^{*} \otimes\left(X^{*} \wedge P^{*}\right) \\
& +\mu X^{*} \otimes\left(X^{*} \wedge Q^{*}\right)+\frac{\lambda}{2} X^{*} \otimes\left(Y^{*} \wedge P^{*}\right)+\mu Y^{*} \otimes\left(Y^{*} \wedge Q^{*}\right),
\end{aligned}
$$

where $\theta=a P^{*}+b Q^{*}, a, b \in \mathbb{R}$.

(b) If $\lambda=2 \mu$, the homogeneous Riemannian structures on $\mathcal{A}_{4}(\lambda, \mu)$ are given by

$$
\begin{aligned}
S= & \theta \otimes\left(X^{*} \wedge Y^{*}\right)+\omega \otimes\left(P^{*} \wedge Q^{*}\right)+\rho \otimes\left(X^{*} \wedge P^{*}\right) \\
& +\sigma \otimes\left(X^{*} \wedge Q^{*}\right)+\sigma \otimes\left(Y^{*} \wedge P^{*}\right)-\rho \otimes\left(Y^{*} \wedge Q^{*}\right),
\end{aligned}
$$

where $\theta, \omega, \rho$, and $\sigma$ are differential 1 -forms on $\mathcal{A}_{4}(\lambda, \mu)$ satisfying

$$
\begin{aligned}
& \widetilde{\nabla} \theta=\widetilde{\nabla} \omega=2 \rho \wedge \sigma+2 \mu\left(X^{*} \otimes \rho+Y^{*} \otimes \sigma\right), \\
& \widetilde{\nabla} \rho=\sigma \wedge(\theta+\omega)+\mu\left(P^{*} \otimes \sigma-X^{*} \otimes(\theta+\omega)\right), \\
& \widetilde{\nabla} \sigma=(\theta+\omega) \wedge \rho-\mu\left(P^{*} \otimes \rho+Y^{*} \otimes(\theta+\omega)\right) .
\end{aligned}
$$

Proof If $S$ is a homogeneous Riemannian structure on $\mathcal{A}_{4}(\lambda, \mu)$ then $S_{A B C}=-S_{A C B}$ for all $A, B, C \in \mathfrak{a}_{4}(\lambda, \mu)$. To determine the conditions on $S$ such that $\widetilde{\nabla} R=0$, where $\widetilde{\nabla}=\nabla-S$, we replace $\left(X_{1}, X_{2}, X_{3}, X_{4}\right)$ in (1.3) by $(X, Y, X, P),(X, Y, Y, Q),(X, Y, Y, P),(X, Y, X, Q)$, and $(X, P, X, Q)$, and, by (2.3) and (2.4) we obtain, respectively,

$$
\begin{array}{ll}
\frac{3 \lambda \mu}{2} S_{Z X Q}+\left(\mu^{2}-\lambda^{2}\right) S_{Z Y P}=\frac{\lambda\left(4 \mu^{2}-\lambda^{2}\right)}{2} X^{*}(Z), & \lambda S_{Z X Q}=2 \mu S_{Z Y P} \\
\left(\lambda^{2}-\mu^{2}\right) S_{Z X P}+\frac{3 \lambda \mu}{2} S_{Z Y Q}=\frac{\lambda\left(4 \mu^{2}-\lambda^{2}\right)}{2} Y^{*}(Z), & \lambda S_{Z Y Q}=-2 \mu S_{Z X P}
\end{array}
$$

and

$$
\left(\lambda^{2}-4 \mu^{2}\right) S_{Z P Q}=2 \mu\left(\lambda^{2}-4 \mu^{2}\right) P^{*}(Z)
$$

From equations (2.8) and (2.9), we have, respectively,

$$
\left(\lambda^{2}-4 \mu^{2}\right) S_{Z Y P}=\frac{\lambda\left(\lambda^{2}-4 \mu^{2}\right)}{2} X^{*}(Z)
$$

and

$$
\left(\lambda^{2}-4 \mu^{2}\right) S_{Z X P}=\frac{\lambda\left(4 \mu^{2}-\lambda^{2}\right)}{2} Y^{*}(Z) .
$$

Then, if $\lambda \neq 2 \mu$, we have

$$
\begin{gathered}
S_{Z X P}=-\frac{\lambda}{2} Y^{*}(Z), \quad S_{Z Y P}=\frac{\lambda}{2} X^{*}(Z), \\
S_{Z X Q}=\mu X^{*}(Z), \quad S_{Z Y Q}=\mu Y^{*}(Z), \quad S_{Z P Q}=2 \mu P^{*}(Z),
\end{gathered}
$$


and, in this case, the condition $\widetilde{\nabla} R=0$ in (1.1) is satisfied if and only if equations (2.10) and (2.11) are satisfied for all $Z \in \mathfrak{a}_{4}(\lambda, \mu)$. If $\lambda=2 \mu$, the condition $\widetilde{\nabla} R=0$ is equivalent to the conditions

$$
S_{Z Y Q}=-S_{Z X P}, \quad S_{Z X Q}=S_{Z Y P}
$$

for all $Z \in \mathfrak{a}_{4}(\lambda, \mu)$.

In any case, we put

$$
\theta(Z)=S_{Z X Y}
$$

for any vector field $Z$ on $\mathcal{A}_{4}(\lambda, \mu)$, and if $\lambda=2 \mu$, we put

$$
\rho(Z)=S_{Z X P}, \quad \sigma(Z)=S_{Z Y P}, \quad \omega(Z)=S_{Z P Q} .
$$

Now, if $\lambda \neq 2 \mu$, from (2.10), (2.11) and (2.13), the (0,3)-tensor field $S$ can be written as (2.5), with $\theta$ a differential 1-form on $\mathcal{A}_{4}(\lambda, \mu)$, and, if $\lambda=2 \mu$, from (2.12), (2.13) and (2.14), the tensor field $S$ can be written as (2.6), where $\theta, \omega, \rho$, and $\sigma$ are differential 1-forms on $\mathcal{A}_{4}(\lambda, \mu)$.

We must now determine the conditions for these 1 -forms such that the condition $\widetilde{\nabla} S=0$ in (1.1) is satisfied. First, if we suppose $\lambda \neq 2 \mu$, by (2.3) and the expression (2.5) for $S$, we have

$$
\widetilde{\nabla} X^{*}=-\left(\frac{\lambda}{2} P^{*}+\theta\right) \otimes Y^{*}, \quad \widetilde{\nabla} Y^{*}=\left(\frac{\lambda}{2} P^{*}+\theta\right) \otimes X^{*}, \quad \widetilde{\nabla} P^{*}=\widetilde{\nabla} Q^{*}=0,
$$

and then we can obtain $\widetilde{\nabla}_{Z} S=\left(\widetilde{\nabla}_{Z} \theta\right) \otimes\left(X^{*} \wedge Y^{*}\right)$ for all $Z \in \mathfrak{a}_{4}(\lambda, \mu)$, hence $\widetilde{\nabla} S=0$ if and only if $\widetilde{\nabla} \theta=0$. If we put $\theta=f X^{*}+g Y^{*}+h P^{*}+k Q^{*}$, where $f, g, h, k$ are differentiable functions on $\mathcal{A}_{4}(\lambda, \mu)$, then by (2.15), the equation $\widetilde{\nabla} \theta=0$ is equivalent to the equations

$$
Z(f)+g\left(\frac{\lambda}{2} P^{*}+\theta\right)(Z)=0, \quad Z(g)-f\left(\frac{\lambda}{2} P^{*}+\theta\right)(Z)=0, \quad Z(h)=Z(k)=0,
$$

for any $Z \in \mathfrak{a}_{4}(\lambda, \mu)$. Replacing $Z$ by $X, Y, P$ and $Q$ in each of the two first equations above and using the structure equations (2.1), it follows that $f=g=0$, and then $\theta=h P^{*}+k Q^{*}$, where $h$ and $k$ are constant functions. This ends the proof of part (a) of the theorem. To finish the proof of part (b), we suppose that $\lambda=2 \mu$. By (2.3) and the expression (2.6) for $S$, we obtain

$$
\begin{aligned}
& \widetilde{\nabla} X^{*}=-\left(\mu P^{*}+\theta\right) \otimes Y^{*}-\left(\mu Y^{*}+\rho\right) \otimes P^{*}+\left(\mu X^{*}-\sigma\right) \otimes Q^{*}, \\
& \widetilde{\nabla} Y^{*}=\left(\mu P^{*}+\theta\right) \otimes X^{*}+\left(\mu X^{*}-\sigma\right) \otimes P^{*}+\left(\mu Y^{*}+\rho\right) \otimes Q^{*}, \\
& \widetilde{\nabla} P^{*}=\left(\mu Y^{*}+\rho\right) \otimes X^{*}+\left(\sigma-\mu X^{*}\right) \otimes Y^{*}+\left(2 \mu P^{*}-\omega\right) \otimes Q^{*}, \\
& \widetilde{\nabla} Q^{*}=\left(\sigma-\mu X^{*}\right) \otimes X^{*}-\left(\mu Y^{*}+\rho\right) \otimes Y^{*}+\left(\omega-2 \mu P^{*}\right) \otimes P^{*},
\end{aligned}
$$

and then a computation shows that the condition $\widetilde{\nabla} S=0$ is equivalent to the conditions (2.7).

If for each $a, b \in \mathbb{R}$, we set $\theta=a P^{*}+b Q^{*}, \omega=2 \mu P^{*}, \rho=-\mu Y^{*}, \sigma=\mu X^{*}$, then $(\theta, \omega, \rho, \sigma)$ is a particular solution of the system of differential equations (2.7). Then, if $\lambda=2 \mu$, the tensor field $S$ given by (2.5) is also a homogeneous Riemannian structure on $\mathcal{A}_{4}(\lambda, \mu)$. Then we have the following corollary.

Corollary 2.2 The tensor field $S$ given by (2.5), with $\theta=a P^{*}+b Q^{*}$, is a homogeneous Riemannian structure on the metric Lie group $\mathcal{A}_{4}(\lambda, \mu)$ for all $\lambda, \mu>0$. 
On the other hand, $(\theta, \omega, \rho, \sigma)=(0,0,0,0)$ is other solution of the system (2.7), and then $S=0$ is a homogeneous Riemannian structure on $\mathcal{A}_{4}(\lambda, \mu)$ if $\lambda=2 \mu$; this is equivalent to say that $\nabla R=0$ and it means that the simply connected complete Riemannian manifold $\mathcal{A}_{4}(2 \mu, \mu)$ is a symmetric space. Then, as an immediate consequence of Theorem 2.1 , we also have the following corollary.

Corollary 2.3 The metric Lie group $\mathcal{A}_{4}(\lambda, \mu)$ is a Riemannian symmetric space if and only if $\lambda=2 \mu$.

Remark 2.4 Irreducible symmetric spaces are Einstein, and in [18] Jensen showed that every 4-dimensional simply connected homogeneous Einstein manifold is symmetric. Actually, in terms of the basis $\{X, Y, P, Q\}$ of $\mathfrak{a}_{4}(\lambda, \mu)$, the Ricci tensor of $\mathcal{A}_{4}(\lambda, \mu)$ can be expressed, for any $\lambda, \mu>0$, as the matrix

$$
\operatorname{Ric}=\operatorname{diag}\left(-\frac{\lambda^{2}}{2}-4 \mu^{2},-\frac{\lambda^{2}}{2}-4 \mu^{2}, \frac{\lambda^{2}}{2}-8 \mu^{2},-6 \mu^{2}\right),
$$

and it is a multiple of the metric if and only if $\lambda=2 \mu$. If $k=\sqrt{6} \mu=\frac{\sqrt{6}}{2} \lambda$, then the Ricci tensor of this symmetric space is $\operatorname{Ric}=-k^{2}\langle$,$\rangle .$

\subsection{Reductive decompositions and simply connected groups of isometries of $\mathcal{A}_{4}(\lambda, \mu)$}

To determine the reductive decompositions associated to the homogeneous Riemannian structures on the metric Lie group $\mathcal{A}_{4}(\lambda, \mu)$, we consider the identity element $o$ of $\mathcal{A}_{4}(\lambda, \mu)$, and put $\mathfrak{m}=T_{o}\left(\mathcal{A}_{4}(\lambda, \mu)\right) \equiv \mathfrak{a}_{4}(\lambda, \mu)$.

For each $a, b \in \mathbb{R}$, the (1,2)-tensor field $S=S^{a, b}$ on $\mathcal{A}_{4}(\lambda, \mu)$ corresponding to the homogeneous Riemannian structure given by (2.5), where $\theta=a P^{*}+b Q^{*}$, is expressed in terms of the basis $\{X, Y, P, Q\}$ of $\mathfrak{a}_{4}(\lambda, \mu)$ by

$$
\begin{array}{llll}
S_{X} X=\mu Q, & S_{Y} X=-\frac{\lambda}{2} P, & S_{P} X=a Y, & \\
S_{X} Y=\frac{\lambda}{2} P, & S_{Y} Y=\mu Q, & S_{P} Y=-a X, & S_{Q} Y=-b X, \\
S_{X} P=-\frac{\lambda}{2} Y, & S_{Y} P=\frac{\lambda}{2} X, & S_{P} P=2 \mu Q, & S_{Q} P=0, \\
S_{X} Q=-\mu X, & S_{Y} Q=-\mu Y, & S_{P} Q=-2 \mu P, & S_{Q} Q=0 .
\end{array}
$$

Then the connection $\widetilde{\nabla}=\widetilde{\nabla}^{a, b}=\nabla-S^{a, b}$ is given by

$$
\widetilde{\nabla}_{P} X=-\left(\frac{\lambda}{2}+a\right) Y, \quad \widetilde{\nabla}_{P} Y=\left(\frac{\lambda}{2}+a\right) X, \quad \widetilde{\nabla}_{Q} X=-b Y, \quad \widetilde{\nabla}_{Q} Y=b X,
$$

with all other covariant derivatives between generators being zero, and the only components of the curvature $\widetilde{R}$ of $\widetilde{\nabla}^{a, b}$ which are not always zero are

$$
\widetilde{R}_{X Y}=\lambda\left(\frac{\lambda}{2}+a\right)\left(Y^{*} \otimes X-X^{*} \otimes Y\right), \quad \widetilde{R}_{P Q}=2 \mu\left(\frac{\lambda}{2}+a\right)\left(X^{*} \otimes Y-Y^{*} \otimes X\right) .
$$

The holonomy algebra $\tilde{\mathfrak{h}}$ of $\widetilde{\nabla}$ is the Lie subalgebra of antisymmetric endomorphisms of $\mathfrak{m} \equiv \mathfrak{a}_{4}(\lambda, \mu)$ generated by the curvature operators $\widetilde{R}_{V W} \in \mathfrak{s o}(\mathfrak{m}) \cong \mathfrak{s o}(4)$, and $\tilde{\mathfrak{g}}^{a, b}=\tilde{\mathfrak{h}}+\mathfrak{m}$, with Lie bracket defined by (1.2), is the reductive decomposition associated to $S=S^{a, b}$. 
If $a=-\lambda / 2$, the holonomy algebra $\tilde{\mathfrak{h}}$ is trivial, and the reductive decomposition associated to the homogeneous Riemannian structure $S=S^{a, b}$ given by (2.5) is $\tilde{\mathfrak{g}}^{b}=\tilde{\mathfrak{g}}^{-\lambda / 2, b}=$ $\{0\}+\mathfrak{m}=\operatorname{Span}\{X, Y, P, Q\}$, with, by (1.2) and (2.17), nonzero brackets

$$
[X, Y]=\lambda P, \quad[Q, X]=\mu X+b Y, \quad[Q, Y]=-b X+\mu Y, \quad[Q, P]=2 \mu P,
$$

that is, $\tilde{\mathfrak{g}}^{b}$ is the semidirect product of the Heisenberg algebra $\mathfrak{h}_{3}$ and the line $\mathfrak{q}=\operatorname{Span}\{Q\}$ under $\delta: \mathfrak{q} \rightarrow \operatorname{Der}\left(\mathfrak{h}_{3}\right)$, where

$$
\delta(Q)=\left(\begin{array}{ccc}
\mu & -b & 0 \\
b & \mu & 0 \\
0 & 0 & 2 \mu
\end{array}\right),
$$

in terms of the basis $\{X, Y, P\}$ of $\mathfrak{h}_{3}$. The simply connected Lie group $\tilde{G}_{\lambda, \mu}^{b}$ generated by $\tilde{\mathfrak{g}}^{b}$ is the semidirect product $H_{3} \rtimes_{\Delta} \mathbb{R}$, where $\Delta: \mathbb{R} \rightarrow \operatorname{Aut}\left(H_{3}\right)$ is the homomorphism given by $\Delta_{t}(z, p)=\left(\mathrm{e}^{(\mu+\mathrm{i} b) t} z, \mathrm{e}^{2 \mu t} p\right)$. Then we have the following theorem.

Theorem 2.5 Let $S=S^{a, b}$ be the homogeneous Riemannian structure on $\mathcal{A}_{4}(\lambda, \mu)$ given by (2.5), and $a=-\lambda / 2$. For each $b \in \mathbb{R}$, the corresponding simply connected group of isometries acting transitively on $\mathcal{A}_{4}(\lambda, \mu)$ is $\tilde{G}_{\lambda, \mu}^{b}=H_{3} \rtimes \mathbb{R}=\mathbb{C} \times \mathbb{R}^{2}$ with the operation given by

$$
(z, p, q) \cdot\left(z^{\prime}, p^{\prime}, q^{\prime}\right)=\left(z+\mathrm{e}^{(\mu+\mathrm{i} b) q} z^{\prime}, p+\mathrm{e}^{2 \mu q} p^{\prime}+\frac{\lambda}{2} \operatorname{Im}\left(\mathrm{e}^{(\mu+\mathrm{i} b) q} \bar{z} z^{\prime}\right), q+q^{\prime}\right) .
$$

Remark 2.6 For each $b \in \mathbb{R}$, the Lie group $\tilde{G}_{\lambda, \mu}^{b}$ associated to $S^{-\frac{\lambda}{2}, b}$ acts simply transitively on $\mathcal{A}_{4}(\lambda, \mu)$, then we have an infinite number of descriptions of $\mathcal{A}_{4}(\lambda, \mu)$ provided by a one-parameter family of non-isomorphic 3-step solvable Lie groups which are extensions of the Heisenberg group. In particular, if $b=0$, the group of isometries is $\mathcal{A}_{4}(\lambda, \mu)$ acting on itself by left translations. In this case, the corresponding homogeneous structure is given by $S^{-\frac{\lambda}{2}, 0}{ }_{Z} W=\nabla_{Z} W$ for all $Z, W \in \mathfrak{a}_{4}(\lambda, \mu)$, which means that $\widetilde{\nabla}^{-\frac{\lambda}{2}, 0}=\nabla-S^{-\frac{\lambda}{2}, 0}$ is the connection on $\mathcal{A}_{4}(\lambda, \mu)$ for which every left-invariant vector field is parallel.

Suppose now that $a \neq-\lambda / 2$. By (2.19), the holonomy algebra $\tilde{\mathfrak{h}}$ of $\widetilde{\nabla}$ is generated by $V=Y^{*} \otimes X-X^{*} \otimes Y \in \mathfrak{s o}(\mathfrak{m})$. Then, by (1.2) and (2.17), the reductive decomposition associated to $S^{a, b}$ is $\tilde{\mathfrak{g}}^{a, b}=\tilde{\mathfrak{h}}+\mathfrak{m}=\operatorname{Span}\{V, X, Y, P, Q\}$, with structure equations

$$
\begin{array}{lll}
{[V, X]=-Y, \quad[V, Y]=X,} & {[V, P]=[V, Q]=0,} \\
{[X, Y]=\lambda\left(\frac{\lambda}{2}+a\right) V+\lambda P,} & {[P, Q]=-2 \mu\left(\frac{\lambda}{2}+a\right) V-2 \mu P,} \\
{[X, P]=-\left(\frac{\lambda}{2}+a\right) Y,} & {[Y, P]=\left(\frac{\lambda}{2}+a\right) X,} \\
{[X, Q]=-\mu X-b Y,} & {[Y, Q]=-\mu Y+b X .}
\end{array}
$$

If we set

$$
\hat{V}=\left(\frac{\lambda}{2}+a\right) V+P, \quad \hat{Q}=Q-b\left(\frac{\lambda}{2}+a\right)^{-1} P, \quad \hat{X}=X, \quad \hat{Y}=Y, \quad \hat{P}=P,
$$

then, with respect to the basis $\{\hat{V}, \hat{X}, \hat{Y}, \hat{P}, \hat{Q}\}$ of $\tilde{\mathfrak{g}}^{a, b}$, the nonzero brackets are

$$
\begin{aligned}
& {[\hat{X}, \hat{Y}]=\lambda \hat{V}, \quad[\hat{Q}, \hat{X}]=\mu \hat{X}, \quad[\hat{Q}, \hat{Y}]=\mu \hat{Y}, \quad[\hat{Q}, \hat{V}]=2 \mu \hat{V},} \\
& {[\hat{P}, \hat{X}]=\left(\frac{\lambda}{2}+a\right) \hat{Y}, \quad[\hat{P}, \hat{Y}]=-\left(\frac{\lambda}{2}+a\right) \hat{X}, \quad[\hat{P}, \hat{Q}]=-2 \mu \hat{V},}
\end{aligned}
$$


that is, $\tilde{\mathfrak{g}}^{a, b}$ is the semidirect product of the Lie algebra $\mathfrak{g}_{0}=\operatorname{Span}\{\hat{X}, \hat{Y}, \hat{V}, \hat{Q}\}$ isomorphic to $\mathfrak{a}_{4}(\lambda, \mu)$ and the line $\mathfrak{p}=\operatorname{Span}\{\hat{P}\}$, with respect to the homomorphism $\delta: \mathfrak{p} \rightarrow \operatorname{Der}\left(\mathfrak{g}_{0}\right)$ given, in terms of the basis $\{\hat{X}, \hat{Y}, \hat{V}, \hat{Q}\}$ of $\mathfrak{g}_{0}$, by

$$
\delta(\hat{P})=\left(\begin{array}{cccc}
0 & -\left(\frac{\lambda}{2}+a\right) & 0 & 0 \\
\frac{\lambda}{2}+a & 0 & 0 & 0 \\
0 & 0 & 0 & -2 \mu \\
0 & 0 & 0 & 0
\end{array}\right)
$$

The simply connected Lie group $\tilde{G}_{\lambda, \mu}^{a, b}$ with Lie algebra $\tilde{\mathfrak{g}}^{a, b}$ is a semidirect product $G_{0} \rtimes_{\Delta} \mathbb{R}$, where we consider $G_{0}$ (which is isomorphic to $\mathcal{A}_{4}(\lambda, \mu)$ ) with global coordinates given by the diffeomorphism

$$
(z=x+\mathrm{i} y, r, s) \in \mathbb{C} \times \mathbb{R}^{2} \rightarrow \exp (x \hat{X}+y \hat{Y}+r \hat{V}) \exp (s \hat{Q}) \in G_{0},
$$

and by using the exponential map for $G_{0}$ (see (2.2)) one has that $\Delta: \mathbb{R} \rightarrow \operatorname{Aut}\left(G_{0}\right)$ is given by $\Delta_{p}(z, r, s)=\left(\mathrm{e}^{\mathrm{i}\left(\frac{\lambda}{2}+a\right) p} z, r+\left(1-\mathrm{e}^{2 \mu s}\right) p, s\right)$. Thus we have the following theorem.

Theorem 2.7 Let $S=S^{a, b}$ be the homogeneous Riemannian structure on $\mathcal{A}_{4}(\lambda, \mu)$ given by (2.5). For each $a, b \in \mathbb{R}, a \neq-\lambda / 2$, the corresponding simply connected group of isometries acting transitively on $\mathcal{A}_{4}(\lambda, \mu)$ is a semidirect product $\mathcal{A}_{4}(\lambda, \mu) \rtimes \mathbb{R}$ and it is isomorphic to $\mathbb{C} \times \mathbb{R}^{3}$ with the group operation

$$
\begin{aligned}
(z, r, s, p) \cdot\left(z^{\prime}, r^{\prime}, s^{\prime}, p^{\prime}\right) & =\left(z+\mathrm{e}^{\mu s+\mathrm{i}\left(\frac{\lambda}{2}+a\right) p} z^{\prime},\right. \\
r & \left.+\mathrm{e}^{2 \mu s}\left(\left(1-\mathrm{e}^{2 \mu s^{\prime}}\right) p+r^{\prime}\right)+\frac{\lambda}{2} \mathrm{e}^{\mu s} \operatorname{Im}\left(\mathrm{e}^{\mathrm{i}\left(\frac{\lambda}{2}+a\right) p} \bar{z} z^{\prime}\right), s+s^{\prime}, p+p^{\prime}\right) .
\end{aligned}
$$

Remark 2.8 If $\lambda=2 \mu$, then $S=0$ is a homogeneous Riemannian structure on $\mathcal{A}_{4}(\lambda, \mu)$, and it describes this manifold as a Riemannian symmetric space. For this structure we have that $\widetilde{R}=R$, and by (2.4) the holonomy algebra $\tilde{\mathfrak{h}}$ of $\widetilde{\nabla}=\nabla$ is the Lie subalgebra of $\mathfrak{s o}(\mathfrak{m})$ generated by

$$
\left(\begin{array}{cccc}
0 & 1 & 0 & 0 \\
-1 & 0 & 0 & 0 \\
0 & 0 & 0 & 0 \\
0 & 0 & 0 & 0
\end{array}\right), \quad\left(\begin{array}{cccc}
0 & 0 & 0 & 0 \\
0 & 0 & 0 & 0 \\
0 & 0 & 0 & 1 \\
0 & 0 & -1 & 0
\end{array}\right), \quad\left(\begin{array}{cccc}
0 & 0 & 1 & 0 \\
0 & 0 & 0 & -1 \\
-1 & 0 & 0 & 0 \\
0 & 1 & 0 & 0
\end{array}\right), \quad\left(\begin{array}{cccc}
0 & 0 & 0 & 1 \\
0 & 0 & 1 & 0 \\
0 & -1 & 0 & 0 \\
-1 & 0 & 0 & 0
\end{array}\right),
$$

which is isomorphic to $\mathfrak{s}(\mathfrak{u}(2) \oplus \mathfrak{u}(1))$. The 8-dimensional Lie algebra $\tilde{\mathfrak{g}}=\tilde{\mathfrak{h}}+\mathfrak{m}$, with the brackets defined by (1.2), is isomorphic to $\mathfrak{s u}(2,1)$, and the reductive decomposition gives the description of $\mathcal{A}_{4}(2 \mu, \mu)$ as the complex hyperbolic plane $\mathrm{SU}(2,1) / \mathrm{S}(\mathrm{U}(2) \times \mathrm{U}(1))=$ $\mathbb{C H}(2)$.

2.3 The Hermitian Lie group $\mathcal{A}_{4}(\lambda, \mu)$

An almost Hermitian manifold $(M, g, J)$ is called a homogeneous almost Hermitian manifold if there exists a Lie group of almost complex isometries acting transitively and effectively on $M$. In [23], Sekigawa proved that a simply connected and complete almost Hermitian manifold $(M, g, J)$ is homogeneous if and only if it admits a tensor field $S$ of type $(1,2)$ satisfying the Ambrose-Singer equations (1.1) and $\widetilde{\nabla} J=0$, where $\widetilde{\nabla}=\nabla-S$. Such a tensor 
field $S$ is called a homogeneous almost Hermitian structure (or a homogeneous Hermitian structure if $(M, g, J)$ is a Hermitian manifold, that is, $J$ is integrable; or a homogeneous Kähler structure if $(M, g, J)$ is Kähler, that is, $J$ is integrable and the fundamental 2-form $\Omega$ on $M$, given by $\Omega(Z, W)=g(Z, J W)$, is closed, or equivalently $\nabla J=0)$. It is well known that $J$ is integrable, that is, it defines a complex structure on $M$, if and only if $N_{J}=0$, where $N_{J}$ is the Nijenhuis tensor, defined by

$$
N_{J}(Z, W)=[J Z, J W]-J[J Z, W]-J[Z, J W]-[Z, W] .
$$

Moreover, a homogeneous Riemannian structure on a Kähler manifold $(M, g, J)$ is a homogeneous Kähler structure if and only if $S \cdot J=0$ or, equivalently, $S_{Z A B}=S_{Z J A J B}$ for all vector fields $A, B, Z$ on $M$.

Suppose that $G$ is a Lie group with the left-invariant Riemannian metric induced by a scalar product $\langle$,$\rangle on its Lie algebra \mathfrak{g}$. An invariant Hermitian structure on the metric Lie group $G$ is an endomorphism $J$ of the Lie algebra $\mathfrak{g}$ of $G$ such that $J^{2}=-\mathrm{id},\langle J A, J B\rangle=$ $\langle A, B\rangle$, and $N_{J}(A, B)=0$ for all $A, B \in \mathfrak{g}$. In this case, the underlying manifold of $G$ is a Hermitian manifold and the left translations on $G$ are holomorphic isometries, and $G$ is called a Hermitian Lie group. If $J$ is an invariant Hermitian structure on $G$ such that

$$
\langle[A, B], J C\rangle+\langle[B, C], J A\rangle+\langle[C, A], J B\rangle=0,
$$

for all $A, B, C \in \mathfrak{g}$, then $(\mathfrak{g},\langle\rangle, J$,$) is called a Kähler algebra, and G$ is a Kähler Lie group. Condition (2.23) is equivalent to $\mathrm{d} \Omega=0$, where $\Omega$ is the Kähler form of $(G,\langle\rangle, J$,$) . In par-$ ticular, solvable Kähler algebras correspond to simply connected homogeneous Kähler manifolds which admit a simply transitive solvable group of holomorphic isometries (see [16]).

We equip the metric Lie algebra $\mathfrak{a}_{4}(\lambda, \mu)$ with the endomorphism $J$ defined by

$$
J X=Y, \quad J Y=-X, \quad J P=-Q, \quad J Q=P .
$$

Then $J$ defines an almost Hermitian structure on $\mathcal{A}_{4}(\lambda, \mu)$ and we have that $N_{J}(A, B)=0$ for all $A, B \in \mathfrak{a}_{4}(\lambda, \mu)$. Thus, $J$ is an invariant Hermitian structure on $\mathcal{A}_{4}(\lambda, \mu)$ for all $\lambda, \mu>0$. However, equation (2.23) is satisfied if and only if $\lambda=2 \mu$.

If $S=S^{a, b}$ is the homogeneous Riemannian structure on $\mathcal{A}_{4}(\lambda, \mu)$ given by (2.5), then $\widetilde{\nabla}=\nabla-S^{a, b}$ is given by (2.18), and it follows that $\widetilde{\nabla} J=0$, then $S^{a, b}$ is a homogeneous Hermitian structure. If $\lambda=2 \mu$ and $S$ is a homogeneous Riemannian structure on the Kähler manifold $\mathcal{A}_{4}(\lambda, \mu)$ given by (2.6), then by (2.24) and equations (2.12), we have $S_{Z A B}=$ $S_{Z J A J B}$ for all $A, B \in \mathfrak{a}_{4}(\lambda, \mu)$, and hence $S$ is a homogeneous Kähler structure.

Then (see also Remark 2.8) we have the following.

Theorem $2.9\left(\mathcal{A}_{4}(\lambda, \mu), J\right)$ is a Hermitian Lie group for all $\lambda, \mu>0$ and it is a Kähler Lie group if and only if $\lambda=2 \mu$. All the homogeneous Riemannian structures on $\mathcal{A}_{4}(\lambda, \mu)$ are homogeneous Hermitian structures and they are homogeneous Kähler structures if $\lambda=$ $2 \mu .\left(\mathcal{A}_{4}(\lambda, \mu), J\right)$ is a Kähler symmetric space if and only if $\lambda=2 \mu$, and, in this case, its description (which corresponds to the homogeneous Kähler structure $S=0$ ) is the complex hyperbolic plane $\mathrm{SU}(2,1) / \mathrm{S}(\mathrm{U}(2) \times \mathrm{U}(1))$.

\section{A solvable two-dimensional extension of the Heisenberg group}

Let $\lambda$ and $\mu$ be positive real numbers and $v \in \mathbb{R}$. We consider the metric solvable Lie algebra $\mathfrak{a}_{5}(\lambda, \mu, v)$ admitting an orthonormal basis $\{X, Y, P, Q, U\}$ with Lie brackets

$$
[X, Y]=\lambda P, \quad[Q, X]=\mu X, \quad[Q, Y]=\mu Y, \quad[Q, P]=2 \mu P, \quad[Q, U]=v U,
$$


and the other zero. It is the semidirect product of the Heisenberg algebra $\mathfrak{h}_{3}=\operatorname{Span}\{X, Y, P\}$ and the either non-abelian (if $v \neq 0$ ) or abelian (if $v=0$ ) Lie algebra $\mathfrak{e}=\operatorname{Span}\{Q, U\}$ under the homomorphism ad $\operatorname{ad}_{\mathfrak{e}}: \mathfrak{e} \rightarrow \operatorname{Der}\left(\mathfrak{h}_{3}\right)$. We denote by $\mathcal{A}_{5}(\lambda, \mu, v)$ the simply connected Riemannian Lie group defined by the metric Lie algebra $\mathfrak{a}_{5}(\lambda, \mu, v)$. Then, $\mathcal{A}_{5}(\lambda, \mu, v)$ can be identified with $\mathbb{C} \times \mathbb{R}^{3}$, with the group operation

$$
(z, p, q, u) \cdot\left(z^{\prime}, p^{\prime}, q^{\prime}, u^{\prime}\right)=\left(z+\mathrm{e}^{\mu q} z^{\prime}, p+\mathrm{e}^{2 \mu q} p^{\prime}+\frac{\lambda}{2} \mathrm{e}^{\mu q} \operatorname{Im}\left(\bar{z} z^{\prime}\right), q+q^{\prime}, u+\mathrm{e}^{v q} u^{\prime}\right),
$$

and the left-invariant metric

$$
\begin{aligned}
g_{\lambda, \mu, v}=\mathrm{e}^{-4 \mu q}\left\{\left(\mathrm{e}^{2 \mu q}+\frac{\lambda^{2}}{4} y^{2}\right) \mathrm{d} x^{2}+\left(\mathrm{e}^{2 \mu q}+\frac{\lambda^{2}}{4} x^{2}\right) \mathrm{d} y^{2}+\mathrm{d} p^{2}\right. \\
\left.-\frac{\lambda^{2}}{2} x y \mathrm{~d} x \mathrm{~d} y+\lambda(y \mathrm{~d} x \mathrm{~d} p-x \mathrm{~d} y \mathrm{~d} p)\right\}+\mathrm{d} q^{2}+\mathrm{e}^{-2 v q} \mathrm{~d} u^{2} .
\end{aligned}
$$

The Lie group exponential map exp: $\mathfrak{a}_{5}(\lambda, \mu, v) \rightarrow \mathcal{A}_{5}(\lambda, \mu, v)$ is given by

$$
\exp (x X+y Y+p P+q Q+u U)= \begin{cases}\left(\frac{e^{\mu q}-1}{\mu q}(x+\mathrm{i} y), \frac{e^{2 \mu q}-1}{2 \mu q} p, q, \frac{e^{v q}-1}{v q} u\right) & \text { if } q \neq 0, \\ (x+\mathrm{i} y, p, 0, u) & \text { if } q=0 .\end{cases}
$$

For arbitrary $(\lambda, \mu, v)$ and $\left(\lambda^{\prime}, \mu^{\prime}, v^{\prime}\right)$, the groups $\mathcal{A}_{5}(\lambda, \mu, v)$ and $\mathcal{A}_{5}\left(\lambda^{\prime}, \mu^{\prime}, v^{\prime}\right)$ are isomorphic if and only if $\mu v^{\prime}=v \mu^{\prime}$, and the corresponding Riemannian metrics $g_{\lambda, \mu, v}$ and $g_{\lambda^{\prime}, \mu^{\prime}, v^{\prime}}$ do not coincide if $(\lambda, \mu, v) \neq\left(\lambda^{\prime}, \mu^{\prime}, v^{\prime}\right)$.

The Levi-Civita connection on $\mathcal{A}_{5}(\lambda, \mu, v)$ is given by equations (2.3) and

$$
\nabla_{U} Q=-v U, \quad \nabla_{U} U=v Q,
$$

with the other covariant derivatives between generators of $\mathfrak{a}_{5}(\lambda, \mu, v)$ involving $U$ being zero. The Riemannian curvature tensor is now given by (2.4) and

$$
\begin{array}{ll}
R_{X U}=\mu v\left(U^{*} \otimes X-X^{*} \otimes U\right), & R_{Y U}=\mu v\left(U^{*} \otimes Y-Y^{*} \otimes U\right), \\
R_{P U}=2 \mu \nu\left(U^{*} \otimes P-P^{*} \otimes U\right), & R_{Q U}=v^{2}\left(U^{*} \otimes Q-Q^{*} \otimes U\right),
\end{array}
$$

where $\left\{X^{*}, Y^{*}, P^{*}, Q^{*}, U^{*}\right\}$ is the basis of invariant 1-forms on $\mathcal{A}_{5}(\lambda, \mu, v)$ dual to $\{X, Y, P$, $Q, U\}$.

\subsection{Homogeneous Riemannian structures on $\mathcal{A}_{5}(\lambda, \mu, v)$}

We now determine the homogeneous Riemannian structures on $\mathcal{A}_{5}(\lambda, \mu, v)$ in terms of the basis $\left\{X^{*}, Y^{*}, P^{*}, Q^{*}, U^{*}\right\}$ of $\mathfrak{a}_{5}(\lambda, \mu, v)^{*}$. If $S$ is a homogeneous Riemannian structure on $\mathcal{A}_{5}(\lambda, \mu, v)$, we know that the condition $\widetilde{\nabla} g=0$ in (1.1) means that the (0,3)-tensor field $S$ is antisymmetric in its second and third arguments. To get the conditions on $S$ in order that $\widetilde{\nabla} R=0$, we also use the Levi-Civita connection $\nabla$ on $\mathcal{A}_{5}(\lambda, \mu, v)$, given by (2.3) and (3.3), and its curvature tensor, given by (2.4) and (3.4). So if we replace $\left(X_{1}, X_{2}, X_{3}, X_{4}\right)$ in (1.3) by $(Y, P, U, Q),(X, P, U, Q),(X, Y, U, Q),(X, Y, P, U)$, we obtain, respectively

$$
S_{Z X U}=0, \quad S_{Z Y U}=0, \quad S_{Z P U}=0, \quad S_{Z Q U}=-v U^{*}(Z),
$$


and replacing $\left(X_{1}, X_{2}, X_{3}, X_{4}\right)$ by $(X, Y, X, Q),(X, Y, Y, Q)$, respectively, we have

$$
\lambda S_{Z Y Q}+2 \mu S_{Z X P}=0, \quad \lambda S_{Z X Q}-2 \mu S_{Z Y P}=0 .
$$

If $v \neq 0$, and we replace $\left(X_{1}, X_{2}, X_{3}, X_{4}\right)$ in (1.3) by $(X, U, P, U),(Y, U, P, U)$, we obtain, respectively,

$$
S_{Z X P}=-\frac{\lambda}{2} Y^{*}(Z), \quad S_{Z Y P}=\frac{\lambda}{2} X^{*}(Z),
$$

and by (3.6) and (3.7), we also have

$$
S_{Z Y Q}=\mu Y^{*}(Z), \quad S_{Z X Q}=\mu X^{*}(Z) .
$$

Then, if $v \neq 0$, by (3.5), (3.7) and (3.8), we can write

$$
\begin{aligned}
S= & \theta \otimes\left(X^{*} \wedge Y^{*}\right)+\omega \otimes\left(P^{*} \wedge Q^{*}\right)-\frac{\lambda}{2} Y^{*} \otimes\left(X^{*} \wedge P^{*}\right)+\mu X^{*} \otimes\left(X^{*} \wedge Q^{*}\right) \\
& +\frac{\lambda}{2} X^{*} \otimes\left(Y^{*} \wedge P^{*}\right)+\mu Y^{*} \otimes\left(Y^{*} \wedge Q^{*}\right)-v U^{*} \otimes\left(Q^{*} \wedge U^{*}\right),
\end{aligned}
$$

where

$$
\theta(Z)=S_{Z X Y}, \quad \omega(Z)=S_{Z P Q}
$$

On the other hand, we have

$$
\left(\widetilde{\nabla}_{Z} S\right)_{W X Y}=\left(\widetilde{\nabla}_{Z} \theta\right)(W), \quad\left(\widetilde{\nabla}_{Z} S\right)_{W X P}=\mu\left(\omega-2 \mu P^{*}\right)(Z) X^{*}(W),
$$

and in particular, if $\widetilde{\nabla} S=0$ then $\omega=2 \mu P^{*}$. A calculation shows that $S$ in (3.9), with $\omega=$ $2 \mu P^{*}$, satisfies $\widetilde{\nabla} R=0$, and it satisfies $\widetilde{\nabla} S=0$ if and only if $\widetilde{\nabla} \theta=0$. Moreover, by using the structure equations (3.1), one obtains that $\widetilde{\nabla} \theta=0$ if and only if $\theta=a P^{*}+b Q^{*}+c U^{*}$, where $a, b, c$ are real numbers, and we conclude with the following theorem.

Theorem 3.1 If $v \neq 0$, all the homogeneous Riemannian structures on the metric Lie group $\mathcal{A}_{5}(\lambda, \mu, v)$ are given by

$$
\begin{aligned}
S= & \theta \otimes\left(X^{*} \wedge Y^{*}\right)+2 \mu P^{*} \otimes\left(P^{*} \wedge Q^{*}\right)-\frac{\lambda}{2} Y^{*} \otimes\left(X^{*} \wedge P^{*}\right)+\mu X^{*} \otimes\left(X^{*} \wedge Q^{*}\right) \\
& +\frac{\lambda}{2} X^{*} \otimes\left(Y^{*} \wedge P^{*}\right)+\mu Y^{*} \otimes\left(Y^{*} \wedge Q^{*}\right)-v U^{*} \otimes\left(Q^{*} \wedge U^{*}\right),
\end{aligned}
$$

where $\theta=a P^{*}+b Q^{*}+c U^{*}, a, b, c \in \mathbb{R}$.

Suppose now that $v=0$. By (3.5) we have $S_{Z} U=0$ for all $Z \in \mathfrak{a}_{5}(\lambda, \mu, 0)$, and by the second equation in (3.3), we also have $\widetilde{\nabla} U=0$ and $\widetilde{\nabla} U^{*}=0$. Then, using the same reasoning as in the proof of Theorem 2.1, we obtain all the homogeneous Riemannian structures in this case, and we can state the following theorem.

Theorem 3.2 (a) If $\lambda \neq 2 \mu$, all the homogeneous Riemannian structures on $\mathcal{A}_{5}(\lambda, \mu, 0)$ are given by (2.5), where $\theta=a P^{*}+b Q^{*}+c U^{*}$, with $a, b, c \in \mathbb{R}$.

(b) If $\lambda=2 \mu$, all the homogeneous Riemannian structures on $\mathcal{A}_{5}(\lambda, \mu, 0)$ are given by (2.6), where $\theta, \omega, \rho$, and $\sigma$ are differential 1 -forms on $\mathcal{A}_{5}(\lambda, \mu, 0)$ satisfying (2.7).

Corollary 3.3 For each $\lambda, \mu, v \in \mathbb{R}, \lambda, \mu>0$, and for each $a, b, c \in \mathbb{R}$, the tensor field $S$ given by (3.10), where $\theta=a P^{*}+b Q^{*}+c U^{*}$, is a homogeneous Riemannian structure on $\mathcal{A}_{5}(\lambda, \mu, v)$. If $v \neq 0$ or $\lambda \neq 2 \mu$, these are all the possible such structures. 
By Theorems 3.1 and 3.2, $S=0$ is a homogeneous Riemannian structure on $\mathcal{A}_{5}(\lambda, \mu, v)$ if and only if $\lambda=2 \mu$ and $v=0$. Then we have the following corollary.

Corollary 3.4 The metric Lie group $\mathcal{A}_{5}(\lambda, \mu, v)$ is a Riemannian symmetric space if and only if $\lambda=2 \mu$ and $v=0$.

Remark 3.5 The metric Lie group $\mathcal{A}_{4}(\lambda, \mu)$ is a symmetric space if and only if $\lambda=2 \mu$ (Corollary 2.3), and it is also Einstein, but the Riemannian symmetric space $\mathcal{A}_{5}(2 \mu, \mu, 0)=$ $\mathcal{A}_{4}(2 \mu, \mu) \times \mathbb{R}$ is not Einstein. For each $\lambda, \mu>0, v \in \mathbb{R}$, the Ricci tensor Ric of $\mathcal{A}_{5}(\lambda, \mu, v)$ is expressed in terms of the basis $\{X, Y, P, Q, U\}$ of $\mathfrak{a}_{5}(\lambda, \mu, v)$ as the matrix

$$
\operatorname{diag}\left(-\frac{\lambda^{2}}{2}-4 \mu^{2}-\mu v,-\frac{\lambda^{2}}{2}-4 \mu^{2}-\mu v, \frac{\lambda^{2}}{2}-8 \mu^{2}-2 \mu v,-6 \mu^{2}-v^{2},-4 \mu v-v^{2}\right),
$$

and it is easy to see that Ric is a multiple of the metric if and only if

$$
\lambda=\frac{\sqrt{6}}{3} k, \quad \mu=\frac{2 \sqrt{33}}{33} k, \quad v=\frac{\sqrt{33}}{11} k, \quad k>0,
$$

and in this case Ric $=-k^{2}\langle$,$\rangle , with k^{2}=6 \mu^{2}+v^{2}$. We notice that for each $k>0$, the metric Lie group

$$
\mathcal{A}_{5}\left(\frac{\sqrt{6}}{3} k, \frac{2 \sqrt{33}}{33} k, \frac{\sqrt{33}}{11} k\right)
$$

is the unique, up to isometries, nonsymmetric Einstein space in the Alekseevsky's classification [2] of homogeneous Einstein spaces (Ric $=-k^{2} g$ ) with nonpositive curvature and dimension $n \leq 5$ (see also Nikonorov [21], where it is proved that each 5-dimensional noncompact homogeneous Einstein manifold is locally isometric to some standard Einstein solvmanifold).

\subsection{Reductive decompositions and simply connected groups of isometries of $\mathcal{A}_{5}(\lambda, \mu, v)$}

By Corollary 3.3, the tensor fields $S$ given by (3.10) are all the homogeneous Riemannian structures on $\mathcal{A}_{5}(\lambda, \mu, v)$ if $v \neq 0$ or $\lambda \neq 2 \mu$, and they are also homogeneous Riemannian structures (but not the only ones) if $v=0$ and $\lambda=2 \mu$. For each $a, b, c \in \mathbb{R}$, let $S=S^{a, b, c}$ be the corresponding (1,2)-tensor field, where $\theta=a P^{*}+b Q^{*}+c U^{*}$. Then, in terms of the basis $\{X, Y, P, Q, U\}$ of $\mathfrak{a}_{5}(\lambda, \mu, v), S$ is given by (2.17) and

$$
S_{U} X=c Y, \quad S_{U} Y=-c X, \quad S_{U} Q=-v U, \quad S_{U} U=v Q
$$

the other components involving $U$ being zero. The connection $\widetilde{\nabla}=\widetilde{\nabla}^{a, b, c}=\nabla-S^{a, b, c}$ is given by (2.18) and

$$
\widetilde{\nabla}_{U} X=-c Y, \quad \widetilde{\nabla}_{U} Y=c X
$$

with the other covariant derivatives between generators vanishing. The components of the curvature $\widetilde{R}$ of $\widetilde{\nabla}^{a, b, c}$ which are not always zero are those given in (2.19) and

$$
\widetilde{R}_{Q U}=c v\left(Y^{*} \otimes X-X^{*} \otimes Y\right) .
$$

We put $\mathfrak{m}=T_{o}\left(\mathcal{A}_{5}(\lambda, \mu, v)\right) \equiv \mathfrak{a}_{5}(\lambda, \mu, v)$, where $o$ is the identity element of $\mathcal{A}_{5}(\lambda, \mu, v)$. The reductive decomposition associated to $S=S^{a, b, c}$ is $\tilde{\mathfrak{g}}^{a, b, c}=\tilde{\mathfrak{h}}+\mathfrak{m}$ with the Lie bracket in (1.2) and $\tilde{\mathfrak{h}}$ being the holonomy algebra of $\widetilde{\nabla}=\widetilde{\nabla}^{a, b, c}$. 
Theorem 3.6 Let $S=S^{a, b, c}$ be the homogeneous Riemannian structure on $\mathcal{A}_{5}(\lambda, \mu, v)$ given by (3.10), $a=-\lambda / 2$, and let $\tilde{G}_{\lambda, \mu, v}^{b, c}$ be the simply connected group of isometries defined by the reductive decomposition associated to $S^{-\frac{\lambda}{2}, b, c}$.

(a) If $c v=0$, the holonomy algebra of $\widetilde{\nabla}^{-\frac{\lambda}{2}, b, c}$ is trivial, and $\tilde{G}_{\lambda, \mu, v}^{b, c}$ is a semidirect product $H_{3} \rtimes E$, where $E$ is isomorphic to the affine group of the line $\operatorname{Aff}(1)$ if $v \neq 0$ and it is the abelian group $\mathbb{R}^{2}$ if $v=0$. The group operation of $\tilde{G}_{\lambda, \mu, v}^{b, c}=\mathbb{C} \times \mathbb{R}^{3}$ is given by

$$
\begin{aligned}
& (z, p, q, u) \cdot\left(z^{\prime}, p^{\prime}, q^{\prime}, u^{\prime}\right) \\
& \quad=\left(z+\mathrm{e}^{\mu q+\mathrm{i}(b q+c u)} z^{\prime}, p+\mathrm{e}^{2 \mu q} p^{\prime}+\frac{\lambda}{2} \mathrm{e}^{\mu q} \operatorname{Im}\left(\mathrm{e}^{\mathrm{i}(b q+c u)} \bar{z} z^{\prime}\right), q+q^{\prime}, u+\mathrm{e}^{v q} u^{\prime}\right) .
\end{aligned}
$$

In particular, $\tilde{G}_{\lambda, \mu, v}^{0,0}=\mathcal{A}_{5}(\lambda, \mu, v)$.

(b) If $c v \neq 0$, the holonomy algebra of $\widetilde{\nabla}^{-\frac{\lambda}{2}, b, c}$ is one-dimensional, and $\tilde{G}_{\lambda, \mu, v}^{b, c}$ is a semidirect product $H_{3} \rtimes F$, where $F$ is isomorphic to $\operatorname{Aff}(1) \times \mathbb{R}$. The group operation of $\tilde{G}_{\lambda, \mu, v}^{b, c}=\mathbb{C} \times \mathbb{R}^{4}$ is given by

$$
\begin{aligned}
& (z, p, q, t, r) \cdot\left(z^{\prime}, p^{\prime}, q^{\prime}, t^{\prime}, r^{\prime}\right) \\
& \quad=\left(z+\mathrm{e}^{\mu q+\mathrm{i}(b q-r)} z^{\prime}, p+\mathrm{e}^{2 \mu q} p^{\prime}+\frac{\lambda}{2} \mathrm{e}^{\mu q} \operatorname{Im}\left(\mathrm{e}^{\mathrm{i}(b q-r)} \bar{z} z^{\prime}\right), q+q^{\prime}, t+\mathrm{e}^{v q} t^{\prime}, r+r^{\prime}\right) .
\end{aligned}
$$

Proof By (1.2), the Lie algebra $\tilde{\mathfrak{g}}^{b, c}=\tilde{\mathfrak{g}}^{-\lambda / 2, b, c}$ is generated by $X, Y, P, Q, U$, and $c v V$, where $V=Y^{*} \otimes X-X^{*} \otimes Y \in \mathfrak{s o}(\mathfrak{m}) \cong \mathfrak{s o}(5)$, and from the expression for $S=S^{a, b, c}$ given in (2.17) and (3.11) and the components of the curvature $\widetilde{R}$ of $\widetilde{\nabla}=\widetilde{\nabla}^{a, b, c}$ given in (3.13) (those in (2.19) vanish), the Lie brackets are

$$
\begin{gathered}
{[X, Y]=\lambda P, \quad[Q, X]=\mu X+b Y, \quad[Q, Y]=-b X+\mu Y, \quad[Q, P]=2 \mu P,} \\
{[X, U]=-c Y, \quad[Y, U]=c X, \quad[Q, U]=v(c V+U),}
\end{gathered}
$$

and

$$
[V, X]=-Y, \quad[V, Y]=X, \quad \text { if } c v \neq 0,
$$

with the rest vanishing.

(a) If $c v=0$ then $\tilde{\mathfrak{g}}^{b, c}=\{0\}+\mathfrak{m}=\operatorname{Span}\{X, Y, P, Q, U\}$ is the semidirect product of the Heisenberg algebra $\mathfrak{h}_{3}=\operatorname{Span}\{X, Y, P\}$ and the either non-abelian (if $v \neq 0, c=0$ ) or abelian (if $v=0$ ) Lie algebra $\mathfrak{e}=\operatorname{Span}\{Q, U\}$ with respect to the homomorphism $\delta: \mathfrak{e} \rightarrow \operatorname{Der}\left(\mathfrak{h}_{3}\right)$ given in terms of $X, Y, P$ by

$$
\delta(Q)=\left(\begin{array}{ccc}
\mu & -b & 0 \\
b & \mu & 0 \\
0 & 0 & 2 \mu
\end{array}\right), \quad \delta(U)=\left(\begin{array}{ccc}
0 & -c & 0 \\
c & 0 & 0 \\
0 & 0 & 0
\end{array}\right) .
$$

The group law of the simply connected Lie group $E$ generated by $\mathfrak{e}$ is given by $(q, u)\left(q^{\prime}, u^{\prime}\right)=$ $\left(q+q^{\prime}, u+\mathrm{e}^{v q} u^{\prime}\right)$. If $v \neq 0, E$ is isomorphic to the (unique) two-dimensional connected nonabelian real Lie group $\operatorname{Aff}(1)$. Now, the group $\tilde{G}_{\lambda, \mu, v}^{a, b}$ is the semidirect product $H_{3} \rtimes_{\Delta} E$, where $\Delta: E \rightarrow \operatorname{Aut}\left(H_{3}\right)$ is given by $\Delta_{(q, u)}(z, p)=\left(\mathrm{e}^{\mu q+\mathrm{i}(b q+c u)} z, e^{2 \mu q} p\right)$.

(b) If $c v \neq 0$ then $\tilde{\mathfrak{g}}^{b, c}=\tilde{\mathfrak{h}}+\mathfrak{m}=\operatorname{Span}\{V, X, Y, P, Q, U\}$, and if we set $T=U+c V$, then $\tilde{\mathfrak{g}}^{b, c}$ can be written as the semidirect product of the Heisenberg algebra $\mathfrak{h}_{3}=\operatorname{Span}\{X, Y, P\}$ 
and the 3-dimensional Lie algebra $\mathfrak{f}=\operatorname{Span}\{Q, T, V\}$ with non-null bracket $[Q, T]=v T$, under the homomorphism $\delta^{\prime}: \mathfrak{f} \rightarrow \operatorname{Der}\left(\mathfrak{h}_{3}\right)$ given, with respect to $X, Y, P$, by

$$
\delta^{\prime}(Q)=\left(\begin{array}{ccc}
\mu & -b & 0 \\
b & \mu & 0 \\
0 & 0 & 2 \mu
\end{array}\right), \quad \delta^{\prime}(T)=0, \quad \delta^{\prime}(V)=\left(\begin{array}{ccc}
0 & 1 & 0 \\
-1 & 0 & 0 \\
0 & 0 & 0
\end{array}\right) .
$$

The group operation of the simply connected Lie group $F$ (isomorphic to $\operatorname{Aff}(1) \times \mathbb{R}$ ) generated by $\mathfrak{f}$ is defined by $(q, t, r)\left(q^{\prime}, t^{\prime}, r^{\prime}\right)=\left(q+q^{\prime}, t+\mathrm{e}^{v q} t^{\prime}, r+r^{\prime}\right)$, and the exponential map exp: $\mathfrak{f} \rightarrow F$ is given by

$$
\exp (q Q+t T+r V)= \begin{cases}\left(q, \frac{e^{v q}-1}{v q} t, r\right) & \text { if } q \neq 0, \\ (0, t, r) & \text { if } q=0,\end{cases}
$$

then we get that the homomorphism $\Delta^{\prime}: F \rightarrow \operatorname{Aut}\left(H_{3}\right)$ induced by $\delta^{\prime}$ is given by $\Delta_{(q, t, r)}^{\prime}(z, p)$ $=\left(\mathrm{e}^{\mu q+\mathrm{i}(b q-r)} z, \mathrm{e}^{2 \mu q} p\right)$, and since $\tilde{G}_{\lambda, \mu, v}^{a, b}$ is in this case the semidirect product $H_{3} \rtimes_{\Delta^{\prime}} F$, the proof concludes.

Theorem 3.7 For each $\lambda, \mu>0, v \in \mathbb{R}$, let $S=S^{a, b, c}$ be the homogeneous Riemannian structure on $\mathcal{A}_{5}(\lambda, \mu, v)$ given by (3.10). For all $a, b \in \mathbb{R}, a \neq-\lambda / 2$, the corresponding simply connected group of isometries acting transitively on $\mathcal{A}_{5}(\lambda, \mu, v)$ is a semidirect product $\mathcal{A}_{5}(\lambda, \mu, v) \rtimes \mathbb{R}$ and it is isomorphic to $\mathbb{C} \times \mathbb{R}^{3}$ with the group operation

$$
\begin{aligned}
& (z, r, s, t, p) \cdot\left(z^{\prime}, r^{\prime}, s^{\prime}, t^{\prime}, p^{\prime}\right)=\left(z+\mathrm{e}^{\mu s+\mathrm{i}\left(\frac{\lambda}{2}+a\right) p} z^{\prime},\right. \\
& \left.r+\mathrm{e}^{2 \mu s}\left(\left(1-\mathrm{e}^{2 \mu s^{\prime}}\right) p+r^{\prime}\right)+\frac{\lambda}{2} \mathrm{e}^{\mu s} \operatorname{Im}\left(\mathrm{e}^{\mathrm{i}\left(\frac{\lambda}{2}+a\right) p} \bar{z} z^{\prime}\right), s+s^{\prime}, t+\mathrm{e}^{v s} t^{\prime}, p+p^{\prime}\right) .
\end{aligned}
$$

Proof By the expression for the curvature $\widetilde{R}$ of $\widetilde{\nabla}=\widetilde{\nabla}^{a, b, c}$ given by (2.19) and (3.13), the holonomy algebra $\tilde{\mathfrak{h}}$ of $\widetilde{\nabla}$ is generated by $V=Y^{*} \otimes X-X^{*} \otimes Y \in \mathfrak{s o}(\mathfrak{m}) \cong \mathfrak{s o}(5)$. By (2.17) and (3.11), which give the components of $S=S^{a, b, c}$, and from (1.2), the reductive decomposition associated to $S$ is $\tilde{\mathfrak{g}}^{a, b, c}=\tilde{\mathfrak{h}}+\mathfrak{m}=\operatorname{Span}\{V, X, Y, P, Q, U\}$, with the Lie bracket defined by equations (2.20) and

$$
[V, U]=0, \quad[X, U]=-c Y, \quad[Y, U]=c X, \quad[P, U]=0, \quad[Q, U]=v(c V+U) .
$$

We set $\hat{V}, \hat{X}, \hat{Y}, \hat{P}, \hat{Q}$ as in (2.21), and $\hat{U}=U+c V$. Then, with respect to the basis $\{\hat{V}, \hat{X}, \hat{Y}, \hat{P}$, $\hat{Q}, \hat{U}\}$ of $\tilde{\mathfrak{g}}^{a, b, c}$, the Lie brackets are given by equations $(2.22)$ and $[\hat{Q}, \hat{U}]=v \hat{U}$, with the other zero. Then $\tilde{\mathfrak{g}}^{a, b, c}$ is the semidirect product of the Lie algebra $\mathfrak{k}_{0}=\operatorname{Span}\{\hat{X}, \hat{Y}, \hat{V}, \hat{Q}, \hat{U}\}$ isomorphic to $\mathfrak{a}_{5}(\lambda, \mu, v)$ and the line $\mathfrak{p}=\operatorname{Span}\{\hat{P}\}$ under $\delta=\operatorname{ad}_{\mid \mathfrak{p}}: \mathfrak{p} \rightarrow \operatorname{Der}\left(\mathfrak{k}_{0}\right)$. We consider the Lie group $K_{0}$ generated by $\mathfrak{k}_{0}$ with coordinates defined by the diffeomorphism

$$
(z=x+\mathrm{i} y, r, s, t) \in \mathbb{C} \times \mathbb{R}^{3} \longrightarrow \exp (x \hat{X}+y \hat{Y}+r \hat{V}) \exp (s \hat{Q}) \exp (t \hat{U}) \in K_{0} .
$$

Then, by using the exponential map (3.2) for $K_{0} \cong \mathcal{A}_{5}(\lambda, \mu, v)$ one obtains easily that the homomorphism $\Delta: \mathbb{R} \rightarrow \operatorname{Aut}\left(K_{0}\right)$ defined by $\delta$ is given by $\Delta_{p}(z, r, s, t)=\left(\mathrm{e}^{\mathrm{i}\left(\frac{\lambda}{2}+a\right) p} z, r+\right.$ $\left.\left(1-\mathrm{e}^{2 \mu s}\right) p, s, t\right)$, and we conclude the proof.

Remark 3.8 If $\lambda=2 \mu$ and $v=0$, then $S=0$ is a homogeneous Riemannian structure on $\mathcal{A}_{5}(\lambda, \mu, v)$, and its associated reductive decomposition defines a 9-dimensional symmetric Lie algebra $\tilde{\mathfrak{g}}=\tilde{\mathfrak{h}}+\mathfrak{m}$, where the holonomy algebra $\tilde{\mathfrak{h}}$ of the connection $\widetilde{\nabla}=\nabla$ is isomorphic to $\mathfrak{s}(\mathfrak{u}(2) \oplus \mathfrak{u}(1))$, and $\tilde{\mathfrak{g}} \cong \mathfrak{s u}(2,1) \oplus \mathbb{R}$. The corresponding description of $\mathcal{A}_{5}(2 \mu, \mu, 0)$ as a Riemannian symmetric space is $(\mathrm{SU}(2,1) \times \mathbb{R}) / \mathrm{S}(\mathrm{U}(2) \times \mathrm{U}(1))=\mathbb{C H}(2) \times \mathbb{R}$. 
3.3 The almost contact metric manifold $\mathcal{A}_{5}(\lambda, \mu, v)$

The odd-dimensional analogues of the almost Hermitian manifolds are the almost contact metric manifolds (see Blair [4,5]). A $(2 n+1)$-dimensional manifold is called an almost contact metric manifold if it is equipped with a quadruple $(\varphi, \xi, \eta, g)$, where $\varphi$ is a tensor field of type $(1,1), \xi$ a vector field, $\eta$ a differential 1 -form, and $g$ a Riemannian metric on $M$ such that

$$
\varphi^{2}=-\mathrm{id}+\eta \otimes \xi, \quad \eta(\xi)=1, \quad g(\varphi Z, \varphi W)=g(Z, W)-\eta(Z) \eta(W),
$$

for every $Z, W \in \mathfrak{X}(M)$. Then $\varphi \xi=0, \eta \circ \varphi=0$, and $\eta(Z)=g(Z, \xi)$ for all $Z \in \mathfrak{X}(M)$. If $\nabla \varphi=0, M$ is called a cosymplectic manifold (see also the recent paper by Fino and Vezzoni [13])

An almost contact metric manifold $(M, \varphi, \xi, \eta, g)$ is called a homogeneous almost contact metric manifold if $(M=G / H, g)$ is a homogeneous Riemannian manifold such that $\varphi$ is invariant (and hence so are $\xi$ and $\eta$ ) under the action of $G$ (see [11,17,20]). From [19] it follows that if $(M, \varphi, \xi, \eta, g)$ is an almost contact metric manifold such that $M$ is simply connected and $g$ is complete then $(M, \varphi, \xi, \eta, g)$ is a homogeneous almost contact metric manifold if and only if there exists a (1,2)-tensor field $S$ on $M$ satisfying (1.1) and $\widetilde{\nabla} \varphi=0$ (and hence $\widetilde{\nabla} \xi=0$ and $\widetilde{\nabla} \eta=0$ ), where $\widetilde{\nabla}=\nabla-S$. Such a tensor field $S$ is called a homogeneous almost contact metric structure (or a homogeneous cosymplectic structure if $M$ is cosymplectic).

Now we define a quadruple $(\varphi, \xi, \eta, g)$ on $\mathcal{A}_{5}(\lambda, \mu, v)$ in terms of the basis $\{X, Y, P$, $Q, U\}$ of $\mathfrak{a}_{5}(\lambda, \mu, v)$ and its dual basis $\left\{X^{*}, Y^{*}, P^{*}, Q^{*}, U^{*}\right\}$. We set

$$
\varphi=X^{*} \otimes Y-Y^{*} \otimes X-P^{*} \otimes Q+Q^{*} \otimes P, \quad \xi=U, \quad \eta=U^{*}, \quad g=g_{\lambda, \mu, v}
$$

where $g_{\lambda, \mu, v}$ is the metric of $\mathcal{A}_{5}(\lambda, \mu, v)$ (for which $\{X, Y, P, Q, U\}$ is orthonormal). Then $(\varphi, \xi, \eta, g)$ satisfies (3.14), and $\mathcal{A}_{5}(\lambda, \mu, v)$ becomes an almost contact metric manifold for all $\lambda, \mu>0, v \in \mathbb{R}$. Moreover, by the equations (2.3) and (3.3) of the Levi-Civita connection on $\mathcal{A}_{5}(\lambda, \mu, v)$, we have that $\nabla \varphi=0$ if and only if $\lambda=2 \mu$ and $v=0$.

Let $S=S^{a, b, c}$ be the homogeneous Riemannian structure on $\mathcal{A}_{5}(\lambda, \mu, v)$ given by (3.10) (see also Corollary 3.3). The connection $\widetilde{\nabla}=\nabla-S^{a, b, c}$ is given by (2.18) and (3.12) and we have $\widetilde{\nabla} \varphi=0$, then $S^{a, b, c}$ is a homogeneous almost contact metric structure. If $\lambda=2 \mu$ and $v=0$, let $S$ be an arbitrary homogeneous Riemannian structure in (b) of Theorem 3.2 on the cosymplectic manifold $\mathcal{A}_{5}(2 \mu, \mu, 0)$, and $\widetilde{\nabla}=\nabla-S$. From the expressions for $\widetilde{\nabla} X, \widetilde{\nabla} Y$, $\widetilde{\nabla} P, \widetilde{\nabla} Q$ (which immediately follow from equations (2.16)), and $\widetilde{\nabla} U=0$, we have $\widetilde{\nabla} \varphi=0$, then $S$ is a homogeneous cosymplectic structure. We can thus state the next result.

Theorem $3.9 \mathcal{A}_{5}(\lambda, \mu, v)$, with $(\varphi, \xi, \eta, g)$ defined by (3.15), is an almost contact metric manifold for all $\lambda, \mu>0, v \in \mathbb{R}$, and it is a cosymplectic manifold if and only if $\lambda=2 \mu$ and $v=0$. All the homogeneous Riemannian structures on $\mathcal{A}_{5}(\lambda, \mu, v)$ are homogeneous almost contact metric structures, and if $\lambda=2 \mu$ and $v=0$ they are homogeneous cosymplectic structures. Moreover, the almost contact metric manifold $\mathcal{A}_{5}(\lambda, \mu, v)$ is a cosymplectic symmetric space if and only if $\lambda=2 \mu$ and $v=0$. The description of $\mathcal{A}_{5}(2 \mu, \mu, 0)$ as a cosymplectic symmetric space (which corresponds to the structure $S=0$ ) is $(\mathrm{SU}(2,1) \times \mathbb{R}) / \mathrm{S}(\mathrm{U}(2) \times \mathrm{U}(1))$. 


\section{References}

1. Abbena, E., Garbiero, S.: Almost-Hermitian homogeneous structures. Proc. Edinb. Math. Soc. (2) 31, 375-395 (1988)

2. Alekseevsky, D.V.: Homogeneous Riemannian spaces of negative curvature. Math. USSR Sbornik 25(1), 87-109 (1975)

3. Ambrose, W., Singer, I.M.: On homogeneous Riemannian manifolds. Duke Math. J. 25, 647-669 (1958)

4. Blair, D.E.: Contact manifolds in Riemannian geometry. Springer, Heidelberg (1976)

5. Blair, D.E.: Riemannian geometry of contact and symplectic manifolds. Birkhäuser, Boston (2002)

6. Cartan, É.: Sur une classe remarquable d'espaces de Riemann. Bull. Soc. Math. France 55, 114-134 (1927)

7. Castrillón López, M., Gadea, P.M., Oubiña, J.A.: Homogeneous quaternionic Kähler structures on $12-$ dimensional Alekseevsky spaces, J. Geom. Phys. 57(10), 2098-2113 (2007)

8. Castrillón López, M., Gadea, P.M., Oubiña, J.A.: Homogeneous quaternionic Kähler structures on eightdimensional non-compact quaternion-Kähler symmetric spaces. Math. Phys. Anal. Geom. 12(1), 47-74 (2009)

9. Castrillón López, M., Gadea, P.M., Swann, A.F.: Homogeneous quaternionic Kähler structures and quaternionic hyperbolic space. Transform. Groups 11(4), 575-608 (2006)

10. Castrillón López, M., Gadea, P.M., Swann, A.F.: Homogeneous structures on real and complex hyperbolic spaces. Illinois J. Math. 53(2), 561-574(2009)

11. Chinea, D., González, C.: An example of an almost cosymplectic homogeneous manifold, in Differential geometry, Peñíscola, 1985, pp. 133-142; Lecture Notes in Mathematics 1209. Springer, Heidelberg (1986)

12. Fino, A.: Almost contact homogeneous structures. Boll. Un. Mat. Ital. (7) 9-A, 299-311 (1995)

13. Fino, A., Vezzoni, L.: Some results on cosymplectic manifolds. Geom. Dedicata 151, 41-58 (2011)

14. Gadea, P.M., Oubiña, J.A.: Homogeneous Riemannian structures on Berger 3-spheres. Proc. Edinb. Math. Soc. (2) 48(2), 375-387 (2005)

15. Gadea, P.M., Oubiña, J.A.: Homogeneous Kähler and Sasakian structures related to complex hyperbolic spaces. Proc. Edinb. Math. Soc. (2) 53(2), 393-413 (2010)

16. Gindikin, G., Piatetskii-Shapiro, I.I., Vinberg, E.B.: Homogeneous Kähler manifolds. C.I.M.E., Edizione Cremonese, Roma (1967)

17. C. González, G., and D. Chinea, D.: Quasi-Sasakian homogeneous structures on the generalized Heisenberg group $H(p, 1)$. Proc. Amer. Math. Soc. 105, 173-184 (1989)

18. Jensen, G.R.: Homogeneous Einstein spaces of dimension four. J. Differential Geom. 3, 309-349 (1969)

19. Kiričenko, V.F.: On homogeneous Riemannian spaces with invariant tensor structures. Soviet Math. Dokl. 21, 734-737 (1980)

20. Koda, T., Watanabe, Y.: Homogeneous almost contact Riemannian manifolds and infinitesimal models. Boll. Un. Mat. Ital. B (7) 11-B, Suppl. fasc. 2, 11-24 (1997)

21. Nikonorov, Yu.G.: Noncompact homogeneous Einstein 5-manifolds. Geom. Dedicata 113(1), 107-143 (2005)

22. Nomizu, K.: Invariant affine connections on homogeneous spaces. Amer. J. Math. 76, 33-65 (1954)

23. Sekigawa, K.: Notes on homogeneous almost Hermitian manifolds. Hokkaido Math. J. 7, 206-213 (1978)

24. Tricerri, F., Vanhecke, L.: Homogeneous structures on Riemannian manifolds. London Math. Soc. Lect. Notes Ser. vol. 83, Cambridge Univ. Press (1983) 